\title{
Lentivirus Vector Purification Using Anion Exchange HPLC Leads to Improved Gene Transfer
}

BioTechniques 34:1074-1080 (May 2003)

Kaoru Yamada, Douglas M. McCarty, Victoria J. Madden, and Christopher E. Walsh University of North Carolina at Chapel Hill, Chapel Hill, NC, USA

\section{ABSTRACT}

Recombinant lentiviral vectors stably transduce both dividing and nondividing cells. Virus pseudotyping with vesicular stomatitis virus envelope $G(V S V-G)$ protein broadens the host range of lentiviral vector and enables vector concentration by ultracentrifugation. However, as a result of virus vector concentration, contaminating protein debris derived from vector-producing cell culture media is toxic to target cells and reduces the transduction efficiency. Here we report a new and rapid technique for purifying lentivirus vector using the strong anion exchange column that significantly improves gene transfer rates. We purified VSV$G$ pseudotyped self-inactivating lentivirus vector and obtained two protein elution peaks (Peak 1 and Peak 2) corresponding to transducing activity. Peak 1 viral particles were 4-8 times more effective in transducing target cells than Peak 2 or non-purified (pre-HPLC) viral particles. We used purified lentivirus vector expressing the human Fanconi anemia group A (FANCA) gene to transduce murine hematopoietic stem/progenitor cells. We observed a consistent 2- to 3-fold increase in gene transfer rates using Peak 1 purified virus compared with nonpurified virus. We conclude that the purification method using the HPLC system provides the highly purified virus vector that reduces cell toxicity and significantly improves gene transfer in primary cells.

\section{INTRODUCTION}

Lentivirus vector based on the human immunodeficiency virus-1 (HIV1) appears to be one of the promising vectors for gene transfer studies $(1,2)$. The advantageous feature of lentivirus vector is the ability of gene transfer and integration into dividing and nondividing cells (3). The current method of lentivirus vector production is based on the co-transfection method with multiple plasmids expressing virus proteins (the gag, pol, rev, and envelope) and the gene of interest in $293 \mathrm{~T}$ cells. To date, considerable effort has been made to improve the safety of lentivirus vector (4). Furthermore, the pseudotyped envelope with vesicular stomatitis virus envelope G (VSV-G) protein broadens the target cell range and can be concentrated by ultracentrifugation, enabling the higher MOI required for the effective gene transfer of primary cells (5). As a consequence, the ultracentrifugation step also concentrates cellular debris, membrane fragments, and denatured proteins derived from culture media of virus-producing cells. This unwarranted material in the crude vector preparation is toxic to target cells, especially primary cells, and may cause immunogenic reactions in experimental animal models by in vivo vector administration. Therefore, to reduce undesirable effects and increase gene transfer efficiency, the purification of virus vector is required. Lentivirus vector purification using cation exchange column chromatography has been reported (6). Generally, a low pH condition is essential to enhance cationic charge on a virus envelope for the efficient virus separation that decreases the stability of membrane proteins. More recently, the gene transfer of primary hematopoietic cells using lentivirus vector purified by a weak anion exchange column was demonstrated (7). However, no characterization of these particles was performed, including the comparison of transduction efficiency with non-purified vector.

In this study, we examined the utility of column chromatography using the HPLC system to purify VSV-G pseudotyped lentivirus vector. It is well known that a poly-cation such as polybrene and protamine sulfate enhances transduction in retrovirus and lentivirus vector systems $(8,9)$. Based on the known electrostatic interaction between the cell surface and virus envelope membrane, we speculated that a strong anion exchange column might be useful to increase the efficiency of virus vector purification. Here we demonstrate that a strong cationic charged matrix enriches for functional lentivirus vector particles and can eliminate contaminating protein and defective particles. The overall result is the decreased cell toxicity and an improvement in gene transfer rates.

\section{MATERIALS AND METHODS}

\section{Plasmid Construct}

The plasmids that express virus protein (gag, pol, and rev; pMDLg/pRRE and pRSV-Rev), vector plasmid containing the sequences of human PGK internal promoter and EGFP [pRRLsin. hPGK.cPPT.CTS.GFPpre (10)], and VSV-G (pMD.G) were kindly provided by Dr. Thomas Dull (Cell Genesys, Foster City, CA, USA). Human PGK, EGFP, and woodchuck hepatitis virus post-transcriptional element (WPRE) 
were removed by restriction enzyme digestion of pRRLsin.hPGK.cPPT. CTS.GFPpre using EcoRI. EF1- $\alpha$ internal promoter (excised from pBudCE4.1 plasmid; Invitrogen, Carlsbad, CA, USA) and a 4.5-kb coding region of Fanconi anemia group A (FANCA) cDNA were ligated into the backbone plasmid to make the $\mathrm{pSIN} / \mathrm{EF} 1-\alpha / \mathrm{FAN}$ CA (Figure 1).

\section{Production of Lentivirus Vector}

The generation of lentivirus vectors was performed by the transfection of $2 \times$ $10^{6} 293 \mathrm{~T}$ cells with each $8 \mu \mathrm{g}$ pMDLg/pRRE, pMD.G, and pRRLsin. hPGK.cPPT.CTS.GFPpre or pSIN/ EF1- $\alpha / F A N C A$ plasmids in addition of $2.5 \mu \mathrm{g}$ pRSV-Rev plasmid using calcium phosphate. After $48 \mathrm{~h}$, the supernatant was harvested and filtered through a $0.45-\mu \mathrm{m}$ pore size filter and then concentrated to $1 / 100$ volume by ultracentrifugation with $50000 \times \mathrm{g}$ at $4^{\circ} \mathrm{C}$ for $2.5 \mathrm{~h}$ using a SW28 rotor (Beckman Coulter, Fullerton, CA, USA) and Sorvall Ultra $80^{\circledR}$ (Kendro Laboratory Products, Newtown, CT, USA). The virus particle pellet was resuspended in PBS and frozen at $-80^{\circ} \mathrm{C}$ until use.

\section{Purification of Lentivirus Vectors by Anion Exchange Column}

The strong anion exchange column ( $5 \mathrm{~mL}$ gel volume column capable of adsorbing $50 \mathrm{mg}$ protein $/ \mathrm{mL}$ column volume; HiTrap ${ }^{\mathrm{TM}} \mathrm{Q}, \mathrm{HP}$, Amersham Biosciences, Piscataway, NJ, USA) was used with the Dynamax HPLC system (Rainin Instrument, Woburn, MA, USA) at $4^{\circ} \mathrm{C}$. Protein elution was monitored with UV absorbency at $280 \mathrm{~nm}$ with Dynamax UV-C (Rainin Instrument).

Before the virus preparation was loaded onto the column, the column was washed with five column volumes (25 $\mathrm{mL}$ ) of starting buffer (PBS adjusted $\mathrm{pH}$ at 7.5) at a flow rate of $5 \mathrm{~mL} / \mathrm{min}$. The column was then washed with the elution buffer (PBS containing $1 \mathrm{M} \mathrm{NaCl}$ ) at $5 \mathrm{~mL} / \mathrm{min}$ for $5 \mathrm{~min}$ and equilibrated with the starting buffer at $5 \mathrm{~mL} / \mathrm{min}$ for 5 min. The 100-fold concentrated virus vector $(0.5 \mathrm{~mL})$ was suspended in the starting buffer adjusted to a total volume of $1-1.5 \mathrm{~mL}$. The virus vector suspension was centrifuged to remove large debris at $6000 \times g$ for $5 \mathrm{~min}$. Virus vector suspension was loaded through a 5 $\mathrm{mL}$ loading capacity injection loop. The protein elution program was designed to include the washing step for $5 \mathrm{~min}$ and salt gradient step $(0-1 \mathrm{M} \mathrm{NaCl})$ for 10 min. A $2.5 \mathrm{~mL} / \mathrm{min}$ of flow rate was applied. In total, 60 fractions were collected ( $1 \mathrm{~mL} /$ tube). Consistently, two protein peaks were obtained and termed as the "Peak 1" and "Peak 2" (Figure 2a). Each fraction was analyzed for virus particle number and transduction efficiency on 293T cells. The vector expressing the FANCA gene was purified using the identical conditions. Desalting was performed with a centrifugal filter device (Centricon ${ }^{\circledR}$ Plus-20, cut-off 100000 molecular weight; Millipore, Bedford, MA, USA). The membrane was sterilized by washing with $70 \%$

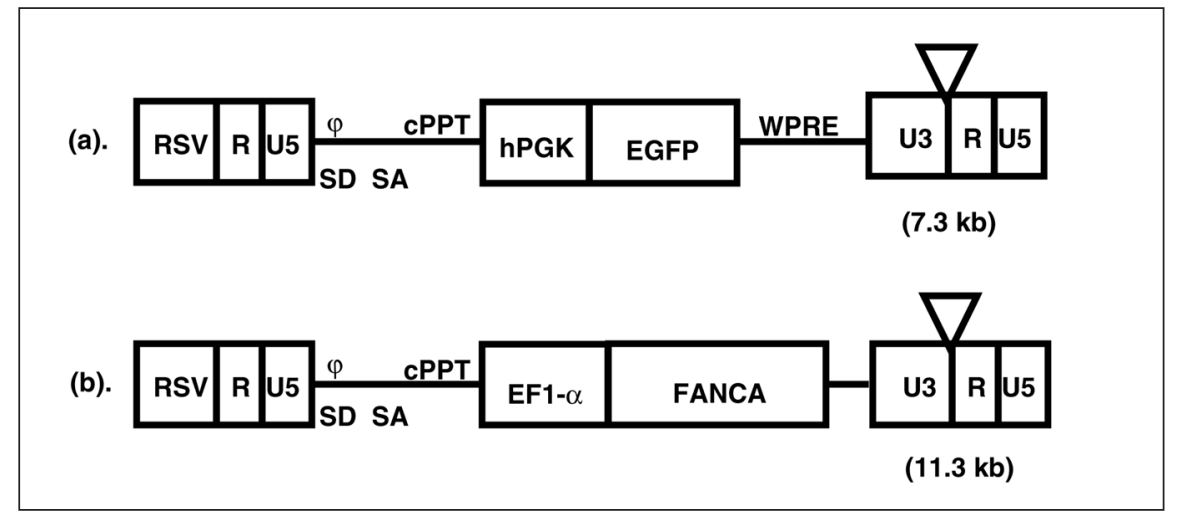

Figure 1. Schematic diagram of vector plasmid constructs. (a) pRRLsin.hPGK.cPPT.CTS.GFPpre. (b) pSIN/EF1- $\alpha$ FANCA. FANCA expression vector (b) was constructed using pRRLsin.hPGK.cPPT.CTS.GFPpre (a). EcoRI restriction enzyme excised the fragment containing hPGK, EGFP, and WPRE. Human elongation factor 1- $\alpha$ promoter and $4.5 \mathrm{~kb}$ FANCA coding sequence were ligated into the backbone plasmid. The triangles represent the deleted sequence in the U3 region.

ethanol. Peak 1 and 2 virus fractions (3-5 fractions) were diluted with $10 \mathrm{~mL}$ PBS and then applied onto the filter device. Virus vector suspensions were centrifuged to desalt and concentrate using a swinging bucket rotor at $2000 \times g$ for $15-20 \mathrm{~min}$ at $4^{\circ} \mathrm{C}$. Virus vector was then recovered from an inverted filter cup with an additional centrifugation step, according to the manufacturer's protocol. The final virus vector volume was adjusted in $200-300 \mu \mathrm{L}$.

\section{Virus Particle Number and EGFP Fluorescence}

Human $293 \mathrm{~T}$ cells were plated into a 48 -well plate at $1 \times 10^{4}$ cells/well and then transduced with EGFP-expressing vector in the presence of $5 \mu \mathrm{g} / \mathrm{mL}$ protamine sulfate. Two days later, EGFP fluorescence was measured using a flow cytometer (FACSCalibur ${ }^{\mathrm{TM}}$; BD Biosciences, San Jose, CA, USA).

For an estimation of the vector particle number in the same volume used for the transduction of 293T cells, virus genomic RNA was harvested as described previously (11). Virus RNA was subjected to dot blot and hybridized using $\alpha$-dCTP ${ }^{32}$-labeled probe generated using the full length of EGFP cDNA (0.6 $\mathrm{kb})$. A standard curve was established using a serial dilution of vector plasmid. After hybridization, the phosphorimage value was captured by a Storm ${ }^{\mathrm{TM}} 860$ phosphorimager (Molecular Dynamics, Sunnyvale, CA, USA). Then, the particle number of each sample was calculated depending on standard curve. The ratio of virus particle number and EGFP fluorescence was determined. The particle number of vector expressing FANCA was also determined using a $1.8-\mathrm{kb}$ fragment of FANCA cDNA (restricted with NheI and BamHI enzymes) as a hybridization probe.

\section{Electron Microscopy}

Virus vector particles were stained using a negative staining technique. A glow discharged, pioloform-coated, 400 mesh nickel grid was placed film-side down onto a $10-\mu \mathrm{L}$ drop of virus vector suspension for $10 \mathrm{~min}$ to allow for particle adsorption. The grid was rinsed briefly by floating on two drops of deionized water and stained by transfer- 
ring it to a drop of $2 \%$ aqueous uranyl acetate $(\mathrm{pH} \mathrm{3.5)}$ for $1 \mathrm{~min}$, followed by air drying. The grids were examined at $80 \mathrm{kV}$ in a LEO EM-910 transmission electron microscope (LEO Electron Microscopy, Thornwood, NY, USA).

\section{Transduction of Mouse Bone}

Clonogenic Assay

Bone marrow was harvested from FANCA knockout mice (kindly provided by Dr. Fre Arwert, Amsterdam, Netherlands). Sca-1 antigen positive cells were selected using the Sca-1 MultiSort kit (Miltenyi Biotech, Auburn, CA, USA) according to the manufacturer's instructions. The cells were incubated with pre-HPLC, and Peak 1 and Peak 2 lentivirus vectors without cytokines were incubated in serum-free media (X-VIVO 10; CAMBREX, Walkersville, MD, USA) for 12
Marrow Hematopoietic Cells and

Table 1. Transduction Efficiency of the Column-Purified Virus

\begin{tabular}{|lcccc|}
\hline Purification & $\begin{array}{c}\text { EGFP-Positive } \\
\text { Cells } \\
(\%)\end{array}$ & $\begin{array}{c}\text { RNA Genome } \\
\text { Particles } \\
\left(\times 10^{5}\right)\end{array}$ & $\begin{array}{c}\text { Transducing Activity } \\
\text { (Genome Particle/ } \\
\text { EGFP-Positive Cell) }\end{array}$ & Fold \\
\hline $\begin{array}{l}\text { Experiment 1 } \\
\text { pre-HPLC }\end{array}$ & 32.8 & 50.3 & $1487: 1$ & 1.0 \\
Peak 1 & 32.8 & 10.6 & $323: 1$ & 4.6 \\
Peak 2 & 15.7 & 12.1 & $770: 1$ & 1.9 \\
Experiment 2 & & & & \\
pre-HPLC & 34.0 & 31.2 & $918: 1$ & 1.0 \\
Peak 1 & 19.2 & 2.1 & $109: 1$ & 8.4 \\
Peak 2 & 12.9 & 4.1 & $314: 1$ & 2.9 \\
\hline
\end{tabular}

h. The minimal MOI (100) needed for mouse bone marrow transduction (12) was used. After transduction, the cells were subjected to clonogenic assay using methylcellulose culture media (MethoCult $^{\mathrm{TM}}$ GF M3534; Stem Cell Technologies, Vancouver, Canada) with serial concentrations of mitomycin $\mathrm{C}$ (a). HPLC profile

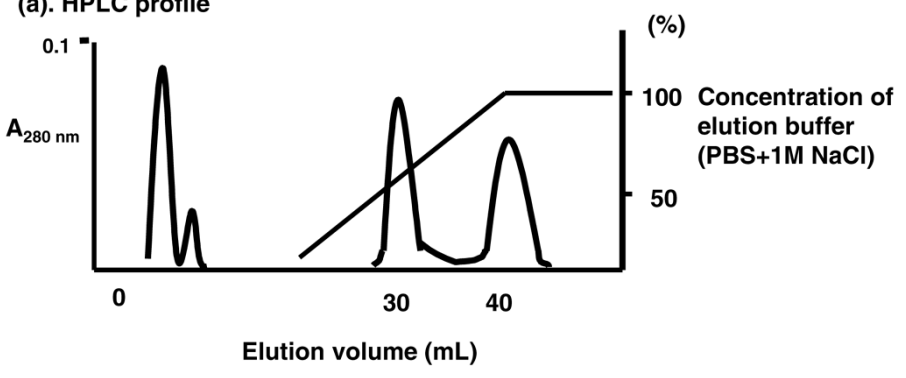

(b). GFP activity

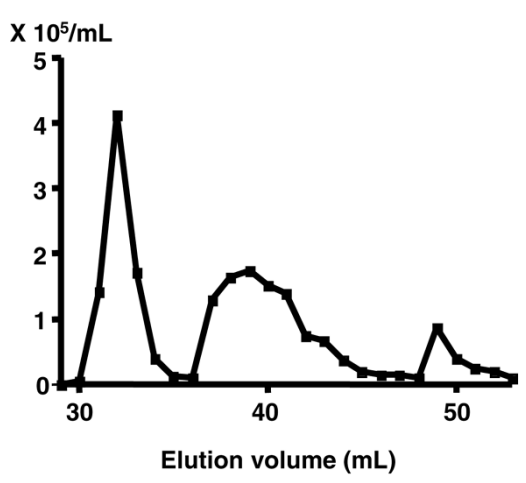

(c). Genome particle number

\section{$X 10^{8} / \mathrm{mL}$}

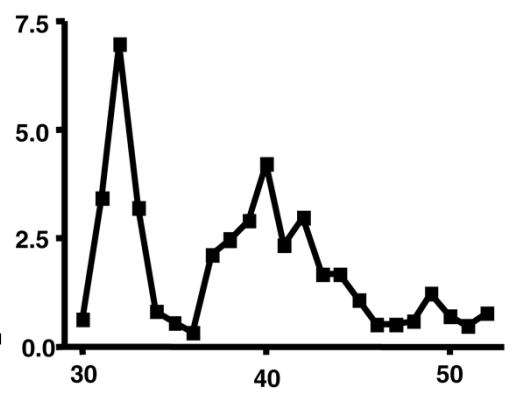

Elution volume $(\mathrm{mL})$
Figure 2. Strong anion exchange column chromatograpy of lentivirus vector. (a) Elution pattern of crude concentrated lentivirus. Virus was applied to the HiTrap Q anion exchange column, washed with PBS, and eluted with PBS containing $1 \mathrm{M} \mathrm{NaCl}$. Fractions of $1 \mathrm{~mL}$ were collected. (b) EGFP activity in cells transduced with eluate. 293T cells $\left(1 \times 10^{4}\right)$ were transduced with of HPLC elution containing lentivirus vector. Two days later, EGFP fluorescence was measured with flow cytometry. Data were expressed as the number of EGFP-positive cells. (c) Genome particle number in cells transduced with eluate. The eluted fractions were treated with DNase I. Virus RNA was extracted and subjected to dot blot analysis using an EGFP probe. The virus particle number was calculated using the standard curve generated with plasmid DNA.
(MMC) (Calbiochem, San Diego, CA, USA) ranging from 0 to $10 \mathrm{nM}$, and the colonies were enumerated 14 days later.

\section{Bone Marrow Transplantation with Transduced Stem/Progenitor Cells}

Lineage-c-kit ${ }^{+} \mathrm{Sca}-1^{+}$cells were isolated from FANCA knockout mice bone marrow following the published method (17) and then transduced with FANCA cDNA expressing vector using the same conditions described above. Twelve hours later after transduction, 3000 cells were injected through orbital vein into sublethally irradiated (4Gy) FANCA knockout mouse. Twenty-four hours after injection, $0.3 \mathrm{mg} / \mathrm{kg}$ MMC was administered intraperitoneally to eliminate nontransduced cells and enrich transgene-expressing cells. After bone marrow transplantation, the blood cell number was monitored weekly. One month later, all blood indices reached normal range (data not shown).

\section{Real-Time PCR}

Peripheral blood $(50 \mu \mathrm{L})$ was collected from recipient mice, and DNA was isolated using the DNeasy ${ }^{\circledR}$ tissue kit (Qiagen, Valencia, CA, USA). Realtime PCR was performed using the ABI PRISM $^{\circledR} 7700$ and GeneAmp ${ }^{\circledR} 5700$ Sequence Detector and software version 1.6.3 (Applied Biosystems, Foster City, CA, USA). The primers and fluorescence-labeled probes specific for the sequence of lentivirus vector (13) and neomycin resistance gene utilized as an internal control were generated using the TaqMan ${ }^{\circledR}$ system (Applied Biosystems). The sequence of the primers and 
probe for neomycin resistance gene are: forward primer 5'-CCATTCGACCACCAAGCG-3', reverse primer 5'-CCGGCTCTCCATCCGA-3', and probe; 5'-TET (tetrachloro-6-carboxyfluorescein)-AACATCGCATCGAGCGAGCACG-TAMRA-3'. A standard curve for the transgene copy number was established by the amplification of a series of genomic DNA mixtures derived from a vector-transduced cell line that has one transgene copy per cell and mouse peripheral blood (data not shown). The quantity of the transgene in the sample DNA was normalized by neomycin resistance gene amplified simultaneously in a separate tube.

\section{RESULTS AND DISCUSSION}

\section{The Purification of Lentivirus} Vector by Anion Exchange Column

The VSV-G pseudotyped virus pellet was resuspended in PBS and loaded onto a HiTrap Q column and eluted with a linear salt gradient (see Materials and Methods section). Consistently, two peaks were obtained after virus elution. The first peak of virus elution appeared when the salt concentration was $0.5 \mathrm{M}$ in PBS (Peak 1, three fractions in total), and the second peak emerges at near $1 \mathrm{M}$ salt (Peak 2, 3-5 fractions). The transduction efficiency of the lentivirus in the eluted fractions was evaluated in the 293T cell line using EGFP fluorescence. The total recovery rate of virus estimated using EGFP fluorescence was $50 \%$ of initial amount of virus loaded onto the column. Two-thirds of the functional particles collected were eluted into Peak 1. The peak fractions of EGFP fluorescence and virus particle number corresponded with the protein elution peak (Figure 2). As demonstrated in Table 1, transducing activity taken as the ratio of EGFP-positive cell number to virus particle number in the same volume of each sample was significantly different between pre-HPLC, Peak 1, and Peak 2 virus. Compared to pre-HPLC virus, column-purified virus based on the transducing activity was 4 - to 8-fold higher in Peak 1 and 2- to 3-fold higher in Peak 2, in the two representative experiments. In other experiments $(n=5)$, we consistently found the similar difference of transduction efficiency between Peak 1 and 2. With electron microscopy, we observed no virus particles aggregating in both Peak 1 and 2 (Figure 3). Because of the high negative charge caused by protein aggregate, a higher salt concentration was required to elute the virus particles into Peak 2. Consequently, the stability of virus envelope may have decreased, resulting in lower transduction efficiency on $293 \mathrm{~T}$ cells. In another experiment, empty viral particles were prepared by transfection without a transgene plasmid, concentrated by ultracentrifuga- tion, and loaded onto the column. A similar elution pattern, including two peaks, was observed. The content of virus genome RNA did not affect the envelope charge, and the empty particles could not be eliminated from the virus preparation (data not shown).

\section{Electron Microscopy}

The pre-HPLC, Peak 1, and Peak 2 virus fractions were examined using an electron microscope after staining with $2 \%$ uranyl acetate. The appearance and size of Peak 1 and Peak 2 virus were not different. The virus size ranged

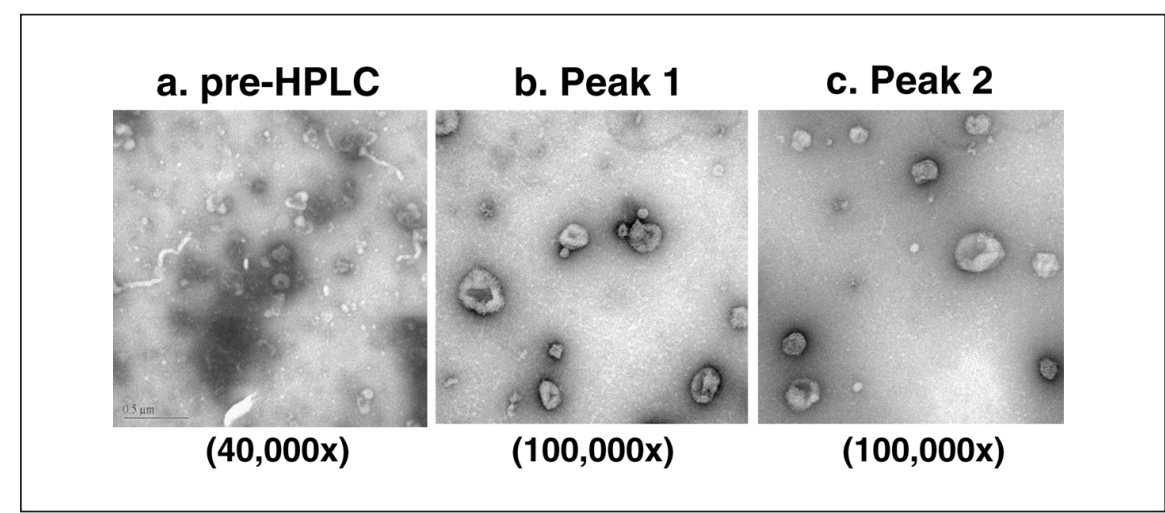

Figure 3. Electron microscopic images of lentivirus particles. Lentivirus preparation was negatively stained with uranyl acetate. (a) Low-magnification (40 000×) electron micrograph of pre-HPLC virus. A significant amount of contaminating protein debris (membrane fragment and proteins) is observed in the concentrated virus preparation. Some virus particles appear as aggregates. (b) High-magnification $(100000 \times)$ picture of Peak 1 virus. (c) Peak 2 virus. The removal of contaminating protein seen in panel a is notable.

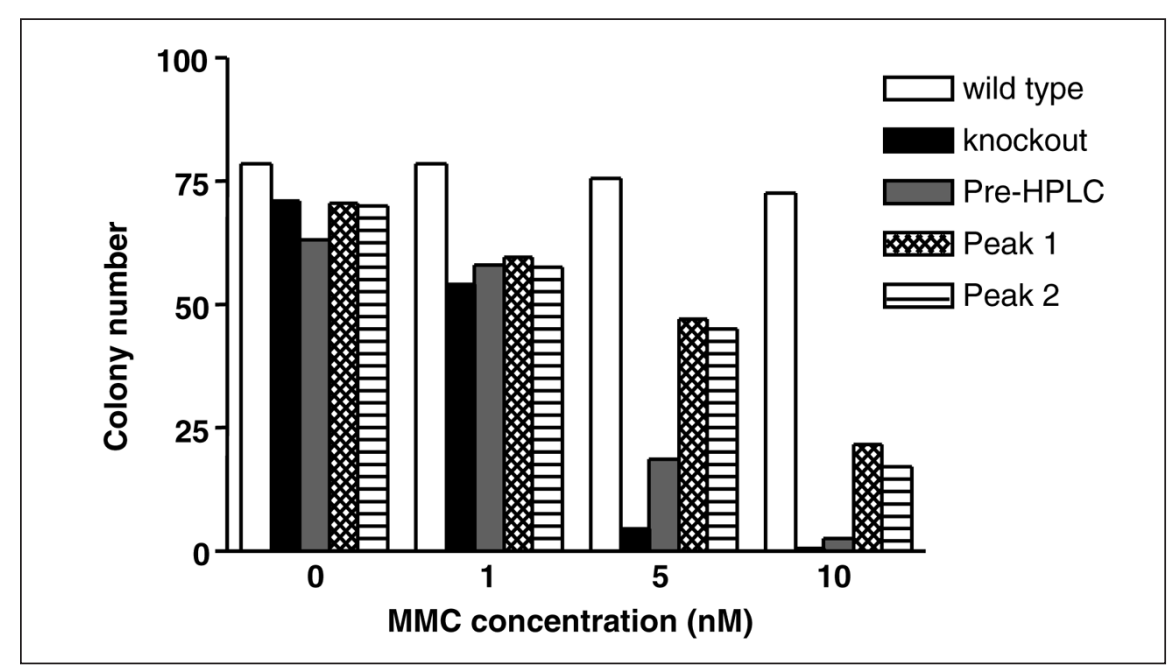

Figure 4. MMC resistance assay of transduced Sca-1 cells. Sca-1 cells were harvested from FANCA wild-type and knockout mouse bone marrow. Knockout Sca-1 cells were transduced with non-purified and column-purified lentivirus vector. After transduction, 1000 cells were subjected to the clonogenic assay in the presence of an increasing concentration of MMC. The colony number designated is the average of duplicate samples. 
from 70 to $130 \mathrm{~nm}$. Because the uranyl acetate also stains the background, in the pre-HPLC sample, we observed significant aggregation of virus particles with protein and cell debris. In Peak 1 and Peak 2 samples, virus particles were observed separately, and the removal of contaminating protein from the background was notable (Figure 3 ).

\section{The Transduction Efficiency of Mouse Bone Marrow}

Fanconi anemia is an autosomal recessive disease characterized by hypersensitivity to alkylating agents such as MMC, which leads to cell death. Cells derived from FANCA knockout mice demonstrate similar sensitivity to MMC. We utilized the human FANCA gene as a biological marker for gene transfer to FANCA knockout mouse bone marrow. First, we harvested Sca-1 positive cells that produce multilineage hematopoietic cells $(14,15)$ from FANCA knockout and wild-type mice bone marrow. After the incubation of Sca-1 cells with each virus preparation, a large amount of protein debris was seen in the serum-free media with pre-HPLC virus, and $90 \%$ of the cells were not viable. On the other hand, much less protein aggregate was observed in the culture with Peak 1 and 2 virus preparations. Transduced cells were subjected to clonogenic assay in the presence of increasing MMC concentrations. As shown in Figure 4, the number of colonies transduced with Peak 1 and Peak 2 virus was 2-fold higher at $5 \mathrm{nM}$ MMC than that of pre-HPLC virus. Unlike EGFP transduction in 293T cells, we did not observe the difference in MMC resistance in bone marrow cells transduced with Peak 1 and Peak 2 virus.

Next, we transduced stem cells from FANCA knockout mice, performed bone marrow transplantation, and then determined proviral integration by realtime PCR. The proviral copy number in the peripheral blood of animals receiving the cells transduced with preHPLC, Peak 1, and Peak 2 virus was $0.34 \pm 0.05,0.73 \pm 0.07 *(P<0.01 *)$, $0.53 \pm 0.10\left(P<0.03^{\#}\right)$, respectively $(*$, \#; compared to pre-HPLC, by paired $t$ test). Peak 1 virus produced a 2 -fold increase of transgene integration in lineage-c-kit ${ }^{+}$Sca- $1^{+}$cells compared with the non-purified virus.

The HPLC lentivirus purification method is a rapid (total processing time is within $30 \mathrm{~min}$ ), simple, and reproducible method for VSV-G pseudotyped virus. It is well known that only less than $1 \%$ of lentivirus vector particles prepared by ultracentrifugation is infectious (13). The RNA genome particle number of vector preparation does not represent the vector infectivity; however, the transducing activity estimated with the ratio of genome particle number and EGFP fluorescence is a better index to determine the functional transgene expression. According to this index, the HPLC virus vector purification technique effectively enriches for functional vector. This is particularly relevant for any potential human clinical gene therapy trials. As indicated in this study, methods to further enrich for functional virus should facilitate better gene transfer rates.

\section{ACKNOWLEDGMENTS}

This work is supported by National Institutes of Health grant RO1 HL 67388, the Fanconi Anemia Research Fund, the American Cancer Society, and the Leukemia and Lymphoma Society to C.E.W.

\section{REFERENCES}

1.Verma, I.M. and N. Somia. 1997. Gene therapy-promises, problems and prospects. Nature 389:239-242

2.Amado, R.G. and I.S. Chen. 1999. Lentiviral vectors - the promise of gene therapy within reach? Science 285:674-676.

3.Uchida, N., R.E. Sutton, A.M. Friera, D. He, M.J. Reitsma, W.C. Chang, G. Veres, R. Scollay, and I.L. Weissman. 1998. HIV, but not murine leukemia virus, vectors mediate high efficiency gene transfer into freshly isolated G0/G1 human hematopoietic stem cells. Proc. Natl. Acad. Sci. USA 95:11939-11944.

4.Dull, T., R. Zufferey, M. Kelly, R.J. Mandel, M. Nguyen, D. Trono, and L. Naldini. 1998. A third-generation lentivirus vector with a conditional packaging system. J. Virol. 72:8463-8471.

5.Burns, J.C., T. Friedmann, W. Driever, M. Burrascano, and J.K. Yee. 1993. Vesicular stomatitis virus $\mathrm{G}$ glycoprotein pseudotyped retroviral vectors: concentration to very high titer and efficient gene transfer into mammalian and nonmammalian cells. Proc. Natl. Acad. Sci. USA 90:8033-8037.

6.Matsuoka, H., K. Miyake, and T. Shimada.
1998. Improved methods of HIV vector mediated gene transfer. Int. J. Hematol. 67:267273.

7.Scherr, M., K. Battmer, U. Blomer, B. Schiedlmeier, A. Ganser, M. Grez, and M. Eder. 2002. Lentiviral gene transfer into peripheral blood-derived CD34+ NOD/SCID-repopulating cells. Blood 99:709-712.

8.Davis, H.E., J.R. Morgan, and M.L. Yarmush. 2002. Polybrene increases retrovirus gene transfer efficiency by enhancing receptor-independent virus adsorption on target cell membranes. Biophys. Chem. 97:159-172. 9.LeDoux, J.M., N. Landazuri, M.L. Yarmush, and J.R. Morgan. 2001. Complexation of retrovirus with cationic and anionic polymers increases the efficiency of gene transfer. Hum. Gene Ther 12:1611-1621.

10.Follenzi, A., L.E. Ailles, S. Bakovic, M. Geuna, and L. Naldini. 2000. Gene transfer by lentiviral vectors is limited by nuclear translocation and rescued by HIV-1 pol sequences. Nat. Genet. 25:217-222.

11.Yamada, K., J.C. Olsen, M. Patel, K.W. Rao, and C.E. Walsh. 2001. Functional correction of Fanconi anemia group C hematopoietic cells by the use of a novel lentiviral vector. Mol. Ther. 3:485-490

12.Chen, W., X. Wu, D.N. Levasseur, H. Liu, L. Lai, J.C. Kappes, and T.M. Townes. 2000 Lentiviral vector transduction of hematopoietic stem cells that mediate long-term reconstitution of lethally irradiated mice. Stem Cells 18:352-359.

13.Scherr, M., K. Battmer, U. Blomer, A. Ganser, and M. Grez. 2001. Quantitative determination of lentiviral vector particle numbers by real-time PCR. BioTechniques 31:520526.

14.Spangrude, G.J., S. Heimfeld, and I.L. Weissman. 1988. Purification and characterization of mouse hematopoietic stem cells. Science 241:58-62.

15.Uchida, N., L. Jerabek, and I.L. Weissman. 1996. Searching for hematopoietic stem cells. II. The heterogeneity of Thy-1.1(lo)Lin(/lo)Sca-1+ mouse hematopoietic stem cells separated by counterflow centrifugal elutriation. Exp. Hematol. 24:649-659.

16.Osawa, M., K. Nakamura, N. Nishi, N. Takahasi, Y. Tokuomoto, H. Inoue, and H. Nakauchi. 1996. In vivo self-renewal of cKit+ Sca-1+ Lin(low/-) hematopoietic stem cells. J. Immunol. 156:3207-3214.

17. Osawa, M., K. Hanada, H. Hamada, and H. Nakauchi. 1996. Long-term lymphohematopoietic reconstitution by a single CD34 low/negative hematopoietic stem cell. Science 273:242-245.

Received 4 November 2002; accepted 22 January 2003.

Address correspondence to:

Dr. Christopher E. Walsh

Mt. Sinai School of Medicine

One Gustave L. Levy Place

Rm. 24-42C Annenberg Bldg.

New York City, NY 10029, USA

e-mail:christopher.e.walsh@mssm.edu 